\title{
ROL DEL FACILITADOR INTERCULTURAL PARA MIGRANTES INTERNACIONALES EN CENTROS DE SALUD CHILENOS: PERSPECTIVAS DE CUATRO GRUPOS DE ACTORES CLAVE
}

\author{
Camila Sepúlveda ${ }^{1, a}$, Báltica Cabieses ${ }^{1,2, b}$
}

\begin{abstract}
RESUMEN
Objetivo. Indagar la percepción de distintos actores clave en torno al rol de facilitadores interculturales en la atención de salud a personas migrantes haitianas en las comunas de Quilicura y Santiago de Chile. Materiales y métodos. Estudio cualitativo de tipo exploratorio y descriptivo, con diseño de estudio de caso, en centros de salud familiar y hospitales de dos comunas de la Región Metropolitana de Chile. Se utilizó la técnica de entrevistas semiestructuradas a actores claves (autoridades sanitarias, trabajadores de salud, facilitadores interculturales y migrantes internacionales), con transcripción verbatim y análisis temático de contenidos $(n=18)$. Resultados. La percepción del rol de los facilitadores interculturales para la atención de salud de migrantes internacionales está relacionada con funciones en traducción, interpretación, educación del sistema sanitario en Chile, mediación intercultural y labores administrativas. Además, colabora en actividades educativas para migrantes que requieren apoyo con el abordaje de diferencias culturales. Esta visión es compartida por diversos actores clave considerados para el estudio y concordante con la política de salud vigente. Conclusión. Los facilitadores interculturales realizan un aporte al encuentro intercultural en salud en Chile y son testigos de cómo nuestras instituciones se enfrentan a realidades diversas en materia lingüística como sociocultural. Reconocer la importancia del facilitador intercultural en la atención sanitaria con pertinencia intercultural hacia población migrante internacional es un avance social y del sistema de salud chileno, que puede ser replicado a países que enfrentan desafíos similares y no desean ignorar la creciente diversidad social y cultural en región de América Latina y el Caribe, como consecuencia de dinámicas transformaciones de procesos de migración internacional.
\end{abstract}

Palabras clave: Migrantes; Salud Pública; Rol Profesional; Competencia Cultural (fuente: DeCS BIREME).

ROLE OF THE INTERCULTURAL FACILITATOR FOR INTERNATIONAL MIGRANTS IN CHILEAN HEALTH CENTRES: PERSPECTIVES FROM FOUR GROUPS OF KEY ACTORS

\begin{abstract}
Objective. To research the perception of different key actors regarding the role of intercultural facilitators in health care for Haitian migrants in the communes of Quilicura and Santiago de Chile. Materials and Methods. Qualitative study of exploratory and descriptive type, with case study design, in family health centers and hospitals of two communes of the Metropolitan Region of Chile. The technique of semi-structured interviews with key actors (health authorities, health workers, intercultural facilitators, and international migrants) was used with verbatim transcription and thematic analysis of contents $(n=18)$. Results. The perception of the role of intercultural facilitators for the health care of international migrants is related to activities like translation, interpretation, health system education in Chile, intercultural mediation, and administrative tasks. In addition, it collaborates in educational activities for migrants who require support in addressing cultural differences. This vision is shared by several key actors considered for the study and according to the current health policy. Conclusions. The intercultural facilitators make a contribution to the intercultural health encounter in Chile, and they are witnesses of how our institutions face different realities in linguistic and socio-cultural matters. Recognizing the importance of the intercultural facilitator in health care with intercultural relevance towards international migrant population is a social advance and one of the Chilean health system, which can be replicated in countries that face similar challenges and do not wish to ignore the growing social and cultural diversity in Latin America and the Caribbean, as a consequence of the dynamic transformations stemming from international migration processes.
\end{abstract}

Keywords: Transients and Migrants; Public Health; Professional Role; Cultural Competency (source: MeSH NLM).

\footnotetext{
Programa de Estudios Sociales en Salud, Facultad de Medicina Clínica Alemana, Universidad del Desarrollo. Santiago, Chile.

Departament of Health Sciences. University of York, York, United Kingdom.

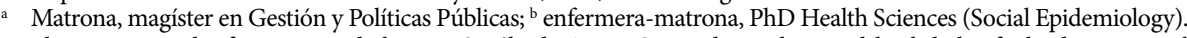

El presente estudio forma parte de la tesis: Sepúlveda-Astete C. Estudio cualitativo del rol de los facilitadores interculturales en la atención de salud de migrantes internacionales en dos comunas de la región Metropolitana: Quilicura y Santiago [tesis para optar al grado de Magíster en Gestión y Políticas Públicas]. Santiago: Facultad de Ciencias Físicas y Matemáticas, Universidad de Chile; 2019.

Recibido: 20/07/2019 Aprobado: 23/10/2019 En línea: 03/12/2019
}

Citar como: Sepúlveda C, Cabieses B. Rol del Facilitador Intercultural para migrantes internacionales en centros de salud chilenos: perspectivas de cuatro grupos de actores clave. Rev Peru Med Exp Salud Publica. 2019;36(4):592-600. doi: 10.17843/rpmesp.2019.364.4683. 


\section{INTRODUCCIÓN}

Aproximadamente un $2,9 \%$ de la población mundial es migrante internacional $(\mathrm{Ml})^{(1)}$. La diversidad de los países de origen es un reto para la adecuada atención de salud en la sociedad receptora, observándose, por ejemplo, barreras administrativas, idiomáticas y culturales ${ }^{(2)}$, que influyen en resultados negativos de salud en estas personas ${ }^{(3)}$.

A nivel internacional, una de las principales barreras en salud en población $\mathrm{Ml}$ es la brecha lingüística, describiéndose la incorporación de traductores e intérpretes en salud ${ }^{(3,4)}$, habitualmente de manera informal como familiares $y / o$ personas voluntarias ${ }^{(5,6)}$. En otros casos, existe incorporación formal de personas que llevan a cabo esta labor en el sistema de salud (7-9). Sobre su efectividad, un estudio en Alemania indica que, aunque en un principio el costo es más alto por la asistencia de intérpretes lingüísticos, a largo plazo estos costos disminuyen, pues permite un tratamiento orientado a los objetivos terapéuticos, evitando la cronicidad y diagnósticos erróneos ${ }^{\left({ }^{10}\right)}$. Otros estudios mencionan que el intérprete transmite aspectos culturalmente específicos, como la comprensión de enfermedad, lo que aumenta la efectividad de los equipos de salud para responder las necesidades particulares del usuario ${ }^{(11,12)}$. Por tanto, el facilitar las condiciones de inclusión de $\mathrm{Ml}$ a los sistemas sanitarios resulta beneficioso, pues una población saludable genera mayor productividad e ingresos para los países receptores ${ }^{(2)}$.

Por otro lado, Chile ha evidenciado un significativo aumento de población $\mathrm{Ml}$, representando actualmente el $6,6 \%$ de la población nacional ${ }^{(13)}$. El $66,7 \%$ de dicha población ingresó entre 2010 y $2017^{(14)}$ con tendencia al alza de personas provenientes de países centroamericanos, cambiando el escenario de las principales colonias de países fronterizos como Argentina, Perú y Bolivia (15). Se observa un aumento marcado de países como Venezuela, con un $23 \%$ del total de $\mathrm{MI}$, sigue Perú con un $17,9 \%$ y Haití con un $14,3 \%{ }^{(13)}$.

La atención a población haitiana es uno de los principales desafíos dentro del sector salud, dadas las barreras idiomáticas y culturales existentes en el encuentro intercultural ${ }^{(16)}$. Los equipos de salud reportan no contar con herramientas suficientes, existiendo limitantes desde el lenguaje, significación de la salud y procesos de enfermedad, hasta aspectos cotidianos como las costumbres alimentarias (17). Para enfrentar esto, los equipos de salud han realizado acciones para mejorar el acceso y cobertura de salud en MI, como capacitaciones sobre interculturalidad en salud, materiales educativos en creol (lengua criolla haitiana) y talleres educativos para MI sobre el funcionamiento del sistema de salud y prestaciones disponibles ${ }^{(18)}$. Además, han incorporado facilitadores interculturales $(\mathrm{FI})$, personas también conocidos como traductores, facilitadores lingüísticos y/o mediadores culturales, dedicados a facilitar la comunicación entre equipo de salud y población migrante haitiana.

\section{MENSAJES CLAVE}

Motivación para realizar el estudio. Debido a las barreras que presentan los migrantes internacionales al acceder a centros de salud en Chile, se creó la figura de facilitador intercultural, pero no se ha estudiado el rol que cumplen.

Principales hallazgos. Se destacan las funciones de los facilitadores en traducción, acogida, orientación, interpretación, entre otras.

Implicancias. Se sugiere revisar la oportunidad que ofrece contar con servicios profesionales de traducción e interpretación en la atención médica, con normativas actualizadas y basadas en la evidencia, abarcando todos los niveles de atención sanitaria.

En Chile, el término $\mathrm{FI}$ se describe por primera vez en el Programa Especial de Salud y Pueblos Indígenas (PESPI) ${ }^{(19)}$, siendo una persona que articula las necesidades de los usuarios con la red de salud y cuyo foco es resolver las inequidades en salud que afectan a personas pertenecientes a estos pueblos ${ }^{(19,20)}$. Con el nuevo escenario migratorio en Chile, se volvió necesario repensar la figura de mediador o facilitador para personas de cultura e idioma diferente, más allá de lo propuesto por el PESPI para pueblos indígenas. Por esta razón, el 2014 se realizaron los primeros esfuerzos para la incorporación del FI para $\mathrm{MI}$ en algunas comunas con alta densidad migratoria, principalmente región Metropolitana $(2,21)$. Considerando estos antecedentes y el progresivo aumento de MI, la Política de Salud de Migrantes Internacionales en Chile ${ }^{(18)}$, incluyó de manera formal la figura de mediadores interculturales y facilitadores lingüísticos. Pese a la relevancia de esta figura en el nuevo escenario social, a la fecha se sabe muy poco sobre el rol de éstos. Por lo cual el objetivo de esta investigación fue indagar la percepción de los distintos actores clave en torno al rol de los facilitadores interculturales en la atención de salud a migrantes haitianos en dos comunas de Chile.

\section{MATERIALES Y MÉTODOS}

Estudio cualitativo de tipo exploratorio y descriptivo, con diseño de estudio de caso, que es considerado apropiado para temas que se consideran prácticamente nuevos, indagando en un fenómeno contemporáneo en su entorno real ${ }^{(22)}$. Este es el primer estudio de su tipo en Chile y su naturaleza cualitativa se justifica cuando el problema de investigación es poco estudiado o no ha sido abordado antes, contribuyendo al diagnóstico basal para estudios posteriores ${ }^{(23)}$.

El estudio fue ejecutado en el 2018 mediante entrevistas semiestructuradas en los niveles de atención primario y secundario. Se realizó en las comunas de Quilicura y Santiago (comunas corresponden a la unidad más básica de administración del Estado de Chile) en la región Metropolitana, que ocupan el primer y tercer lugar en

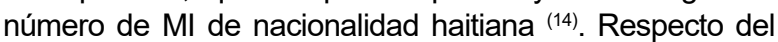
nivel primario, Quilicura tiene a 9627 haitianos inscritos en 
sus centros de salud y tiene cinco FI. Santiago tiene a 2562 haitianos inscritos en sus centros de salud con un $\mathrm{FI}$, que esta físicamente instalado en el Centro de Salud Familiar (CESFAM).

En atención secundaria se trabajó con el Complejo Hospitalario San José, donde la cuarta parte de los partos son de mujeres de nacionalidad haitiana ${ }^{(15)}$. Aquí la primera FI fue contratada en el 2014 y cuentan actualmente con seis $\mathrm{FI}$, transformándose en el único hospital que tiene FI las 24 horas del día. Se trabajó también con el Hospital Clínico San Borja Arriarán, que atiende un alto número de mujeres de nacionalidad haitiana, muchas no hispanoparlantes. La primera $\mathrm{FI}$ fue en el 2016 y actualmente cuentan con dos $\mathrm{FI}$ en horario diurno.

\section{SELECCIÓN DE LA MUESTRA}

La muestra fue intencionada con el propósito de entrevistar a personas que han participado directa o indirectamente en el proceso de construcción y constitución de la figura FI para MI. Se consideraron para este estudio cuatro grupos de actores clave: 1) Autoridad/Referente de migración (AS), 2) Trabajador de Salud (ST), 3) Facilitador Intercultural (FI), y 4) Migrante Internacional (MI), realizándose un total de 18 entrevistas, cuatro de ellas en creol para grupo de MI, lográndose la saturación de la información.

\section{RECOPILACIÓN DE LA INFORMACIÓN}

La investigadora principal acudió a cada centro de salud, previa realización de las entrevistas, para reconocer el funcionamiento de cada centro. Sin embargo, la investigadora, quien habla español y creol, y los participantes del estudio se conocieron el día en que se realizó la entrevista. Éstas fueron ejecutadas en una oficina en cada centro de salud y tuvieron una duración total entre 21 y 44 minutos, contando con registro de audio. Los audios fueron transcritos literalmente y para garantizar confidencialidad se asignaron códigos únicos. El material fue analizado mediante la estrategia de análisis temático de manera integrada para el total de entrevistas ${ }^{(24)}$.

\section{CONSIDERACIONES ÉTICAS}

El proyecto contó con la aprobación del Comité Ético Científico de la Facultad de Medicina Clínica Alemana Universidad del Desarrollo, Comité Ético Científico del Servicio de Salud Metropolitano Central y Comité de Ética de la Investigación del Servicio de Salud Metropolitano Norte. Todos los participantes recibieron información del estudio, en español o creol, firmando un consentimiento informado antes de iniciar su participación en el estudio y recibieron un resumen de los principales resultados del estudio. Para el grupo clave de personas migrantes internacionales que no hablaban español, se garantizó que la gramática empleada en la información del estudio, consentimiento informado, entrevistas y resultados fuera comprensible mediante la traducción, edición según las correcciones de dos nativos y posterior revisión final con otro nativo.

\section{RESULTADOS}

La Tabla 1 describe los participantes del estudio. En cuanto a las áreas académicas de cada grupo de actor clave se distinguen para AS área de salud (psicólogas, trabajadores sociales, nutricionistas y matronas) y área social (socióloga). El grupo de TS participaron profesionales clínicos en salud como: matronas, enfermeras y trabajadores sociales. Los FI tenían profesiones de técnicos en enfermería, recursos humanos y administración. Y los MI con títulos profesionales de ciencias económicas e informática y dos con estudios secundarios.

Los hallazgos fueron agrupados en cinco ejes temáticos: 1) Contexto para la incorporación, 2) Funciones, 3) Ventajas y desventajas del FI, 4) Barreras y facilitadores para incorporar al FI, y 5) Recomendaciones. Dentro de esta última se realizan recomendaciones tanto para la incorporación formal de los FI en los centros de salud como también en torno a la importancia de capacitación en interculturalidad y migración a los equipos de salud. A continuación, se expone la síntesis de los hallazgos agrupados en categorías (Tabla 2).

Tabla 1. Descripción sociodemográfica de los participantes del estudio.

\begin{tabular}{|c|c|c|c|c|c|}
\hline $\begin{array}{l}\text { Características / Grupo de } \\
\text { actores clave }\end{array}$ & $\begin{array}{l}\text { Autoridad sanitaria o } \\
\text { referente de migrantes }\end{array}$ & $\begin{array}{c}\text { Trabajadores de la } \\
\text { salud }\end{array}$ & $\begin{array}{l}\text { Facilitadores } \\
\text { interculturales }\end{array}$ & $\begin{array}{c}\text { Migrantes } \\
\text { internacionales }\end{array}$ & Total \\
\hline \multicolumn{6}{|l|}{ Sexo } \\
\hline Hombre & 3 & 0 & 2 & 0 & 4 \\
\hline Mujer & 2 & 5 & 2 & 4 & 14 \\
\hline Edad (promedio en años) & 42,8 & 31,2 & 29,5 & 33,5 & 34,6 \\
\hline \multicolumn{6}{|l|}{ Nacionalidad } \\
\hline Chilena & 5 & 5 & 0 & 0 & 10 \\
\hline Haitiana & 0 & 0 & 4 & 4 & 8 \\
\hline \multicolumn{6}{|l|}{ Nivel educativo } \\
\hline Secundaria completa & 0 & 0 & 0 & 2 & 2 \\
\hline Técnico profesional & 0 & 0 & 2 & 0 & 2 \\
\hline Universitaria completa & 2 & 4 & 2 & 2 & 10 \\
\hline Posgrado & 3 & 1 & 0 & 0 & 4 \\
\hline
\end{tabular}


Tabla 2. Descripción de guía de entrevistas semiestructuradas, categorías, códigos emergentes y viñetas de descripción de cada código identificado en el estudio.

\begin{tabular}{|c|c|c|c|}
\hline $\begin{array}{l}\text { Guía de } \\
\text { entrevistas: } \\
\text { ¿qué se preguntó? }\end{array}$ & Categorías & $\begin{array}{l}\text { Códigos } \\
\text { emergentes }\end{array}$ & Citas \\
\hline $\begin{array}{l}\text { ¿Qué necesidades } \\
\text { tenían, cómo } \\
\text { ha sido la } \\
\text { experiencia? }\end{array}$ & $\begin{array}{l}\text { Experiencia de } \\
\text { facilitadores } \\
\text { interculturales. }\end{array}$ & $\begin{array}{l}\text { Perfil del facilitador } \\
\text { intercultural. }\end{array}$ & $\begin{array}{l}\text { «Nosotros empezamos a tener fallecimiento de los niños de madres haitianas por falta de } \\
\text { entendimiento de las indicaciones que se les daba, sobre todo de movimientos fetales» } \\
\text { (TS, hospital). } \\
\text { "Una de las acciones principales es la contratación de facilitadores interculturales, porque } \\
\text { de alguna u otra forma cuando hablamos de población migrante hablamos de la población } \\
\text { migrante en general, pero directamente por una base cultural y de brecha idiomática, } \\
\text { directamente nosotros apuntamos a nuestra población de nacionalidad haitiana» (AS, } \\
\text { hospital). } \\
\text { "lgual, la persona que nosotros tenemos es una persona formada en salud (...) entonces, } \\
\text { eso en alguna medida es un plus para que pueda orientar en salud a los pacientes que se } \\
\text { atienden» (TS, CESFAM). } \\
\text { "(...) él tenia cero conocimientos de la parte científica-médica, pero él era una persona } \\
\text { bastante proactiva, llegaba y recordaba todos los conceptos que él habia tenido que } \\
\text { explicar de alguna manera y llegaba al computador y empezaba a investigar qué era una } \\
\text { cesárea, que era una epidural, que era un parto podálico, etcétera» (AS, hospital). }\end{array}$ \\
\hline
\end{tabular}

¿Cuáles son las funciones? ¿Para qué actividades fue contratado?
- Acogida.

- Traducción.

- Interpretación

Descripción de - Educación del funciones realizadas sistema de salud. por los facilitadores - Enlace con la interculturales.

comunidad.
- Orientación.

"...he sacado brechas entre pacientes y profesionales, porque la idea es sacar esas barreras entre profesionales y pacientes, y para los pacientes eso es importante. Entonces, al no entender o comprender, el por qué viene un paciente, es complejo» (FI, hospital).

«En primera instancia, la acogida, es la puerta de entrada de acogida a nuestros vecinos y vecinas migrantes (...) la acogida es la base fundamental, porque en la acogida empieza la instancia de confianza, motivacional, para que también nuestro vecino migrante vuelva a su próximo control o sus próximas atenciones. Y también se abra frente a sus necesidades de salud, obviamente» (AS, CESFAM).

«Las principales funciones son interpretar (...). Ellos hacen todo un proceso de acomodar a su cultura y nos ayudan a nosotros también entender desde el punto de vista cultural» (AS, hospital).

- Promoción de

salud.

- Mediación cultural.

- Administrativa.

«Orientarlos, porque a veces pasaba que tu tratabas de entregarles las indicaciones, pero finalmente ellos se iban, ejemplo con los controles de niños no sabian que tenian que esperar las vacunas (...) La FI ha hecho todo un trabajo de seguirlos post control y eso ha sido importante para darle continuidad a las atenciones» (TS, CESFAM).

«Hay varias cosas distintas que ellos no entienden, que no son iguales acá en Chile, es una cultura diferente, en el caso de las mujeres embarazadas tienen que iniciar sus controles en las primeras semanas de embarazo, pero allá no es así» (FI, CESFAM).

"Los facilitadores se han ido incorporando a la cultura hospitalaria, se han ido adaptando súper bien, son súper queridos dentro del hospital. (...) nosotros hicimos un ciclo, con los facilitadores, conversatorio, para los funcionarios, justamente con esta intención de adaptar, incluir, a los facilitadores y la cultura haitiana y fue una experiencia súper bonita, porque nos contaron de su cultura, de su comida, de sus creencias, bailes, nos hicieron comida» (AS, hospital)

"Cuando saben que la visita se va a hacer con él para ellos es súper aliviador, porque nos estresa hacer una visita a domicilio o atención en box sin nadie que nos pueda ayudar en la traducción» (TS, CESFAM).

- Disminución de gasto público. - Confidencialidad.

AS y TS:

- Comunicación efectiva.

¿Que ventajas y desventajas tiene poder realizar una atención de salud con un facilitador Ventajas y - Uso eficiente de la desventajas de contar red de salud. con facilitadores - Reducción estrés interculturales.
- Contribucio salud. - Contribución a asentar necesidad de capacitación intercultural.
"He notado que a veces, cuando es un hombre que esta con su pareja mujer y yo le doy una indicación, estoy trasmitiendo las indicaciones y le digo al esposo que me traduzca y se lo diga a la mujer, entonces el caballero me queda mirando como "ya, ya" y tengo que repetirle a él que por favor le explique a la señora lo que acabo de decir, y ahi recién como que el empieza a explicarle a la señora, porque si no en el fondo es como que se guardara las indicaciones para él» (TS, hospital).

«Muchas veces el doctor da indicaciones, uno no habla el idioma del doctor, uno no entiende. Por eso, es muy importante que los migrantes puedan encontrar una persona que hable el idioma (...) sobre todo la gente que tiene bebes, que quiere explicar al doctor si el niño tuvo un problema durante la noche» (MI, hospital).

«Ventajas para mi es un buen aporte, por ej. cuando vengo al médico y no hablo español, psicológicamente eso me afecta, pero si yo veo a alquien en el centro que es de mi país, mi mentalidad cambia, entonces esa persona tiene más confianza para poder comunicarse, expresar que es lo que siente y también esa persona se siente como en su cultura, en su país natal» (FI, CESFAM).

«Se les está enseñando creol, pero a la vez la cultura y se hacen poco los cupos, cada vez hay más demanda» (AS, hospital).

TS: Trabajador de Salud; AS: Autoridad/Referente de migración; CESFAM: Centro de Salud Familiar; FI: Facilitador intercultural.

(Continuá en la página 596) 
Tabla 2. Descripción de guía de entrevistas semiestructuradas, categorías, códigos emergentes y ejemplo de viñetas de descripción de cada código identificado en el estudio. (Viene de la página 595)

\begin{tabular}{|c|c|c|c|}
\hline $\begin{array}{l}\text { Guía de } \\
\text { entrevistas: } \\
\text { ¿qué se preguntó? }\end{array}$ & Categorías & $\begin{array}{c}\text { Códigos } \\
\text { emergentes }\end{array}$ & Citas \\
\hline $\begin{array}{l}\text { ¿Cuáles fueron } \\
\text { los principales } \\
\text { problemas y } \\
\text { situaciones que } \\
\text { favorecieron la } \\
\text { inserción del FI? }\end{array}$ & $\begin{array}{c}\text { Barreras y } \\
\text { facilitadores para la } \\
\text { llegada de la figura de } \\
\text { FI en los centros de } \\
\text { salud. }\end{array}$ & $\begin{array}{l}\text { Barreras: } \\
\text { - Administrativa- } \\
\text { financiera. } \\
\text { - Diferencia entre } \\
\text { sistema de salud } \\
\text { chileno y haitiano. }\end{array}$ & $\begin{array}{l}\text { «Más que nada la dificultad administrativa. Te comentaba que no habia precedentes de } \\
\text { contratos de extranjeros no nacionalizados, sin estudios validados, en la administración } \\
\text { pública (...) Fue algo que aqui se hizo, se armó, que no teniamos ninguna experiencia } \\
\text { previa, pero que se ha ido sosteniendo en el tiempo (...) Tuvimos que hacer algunos } \\
\text { escritos, algunas gestiones de que no habia ningún chileno que hablara creol ni conociera } \\
\text { la cultura al menos sanitaria para prestar este servicio, para que finalmente se aprobara } \\
\text { del departamento de juridica, con resolución y todo lo demás para su contratación, si no } \\
\text { fue así tan simple» (AS, hospital). } \\
\text { «Nunca entendieron que las personas que necesitaban también atención eran aquellos } \\
\text { que llegaban a este hospital para que se les informara que tenian que ser operados, que } \\
\text { su enfermedad era irreversible» (AS, hospital). } \\
\text { "...muchas veces no saben relacionarse por diferencias entre salud primaria y urgencia, } \\
\text { entonces, la persona tiene un dolor de cabeza y viene a urgencia, le duele un dedo y viene } \\
\text { también a urgencia, entonces hay profesionales que se molestan y dicen 'Ay, viene a puro } \\
\text { llenarnos'» (FI, hospital). }\end{array}$ \\
\hline & & $\begin{array}{l}\text { Facilitadores: } \\
\text { - Voluntad de } \\
\text { autoridades. } \\
\text { - Identificación } \\
\text { / uniforme } \\
\text { institucional. }\end{array}$ & $\begin{array}{l}\text { "...él (director) tenia una gran sensibilidad con el tema, por lo tanto, no costó nada (...) } \\
\text { Él quería que este hospital, y lo ponía así, como un hospital sin muros, donde eran todos } \\
\text { bienvenidos. Entonces, con ese tipo de director, con esa sensibilidad, no tienes ningún } \\
\text { problema» (AS, hospital). } \\
\text { "...integrándolos al senvicio de salud (...) tiene un uniforme, tiene su credencial, el hospital } \\
\text { saben que ella está presente, sabe el número para llamarla, sabe dónde ubicarla» (TS, hospital). }\end{array}$ \\
\hline
\end{tabular}

"...nosotros tenemos es un folleto que está escrito en creol para las indicaciones del alta, pero hay muchos pacientes haitianos que no necesariamente saben leer, entonces, muchos de ellos se manejan en el creol como idioma hablando, pero no escrito» (TS, hospital).

"Yo creo que en este minuto necesitamos más recurso humano, en primera instancia. Una facilitadora lingüística, como te digo, requiere bastante contención ella misma por toda la demanda de atención que tiene» (AS, hospital).

¿Qué

recomendaciones podría dar para mejorar?
- Contratación de más $\mathrm{FI}$

- Sensibilización del Recomendaciones usuario interno. para la incorporación - Evaluar necesidad de facilitadores interculturales. según realidad local. - Incorporación de FI a las reuniones de sector y estudios de familia. "...hemos solicitado uno o dos facilitadores más para cubrir realmente las necesidades y esto nos ayudaría a mejorar la atención, porque a veces nosotros no estamos en un servicio y nos llaman y no podemos ir hasta media hora más. O sea, aún somos insuficientes» (FI, hospital).

"...lo que yo sugiero para esas comunas con esta mirada migratoria, primero abrirse a recoger otras experiencias primero y desde esa experiencia reorganizarse para poder brindar una mejor acogida. Pero para eso se requieren las voluntades, porque sin las voluntades uno no puede desarrollar ni formar ni crear absolutamente nada» (AS, CESFAM).

«Este facilitador no tiene que ser solamente para traducir, tienen que incorporarse con el equipo y ser de ayuda como un nexo entre la comunidad migrante haitiana y el centro de salud» (AS, CESFAM).

«Capacitarnos más a nosotras como profesionales de salud (...) porque las barreras culturales se quitan solamente con la educación» (TS, hospital).

"No tiene que ser solamente para traducir, tienen que incorporarse con el equipo y ser de ayuda como un nexo entre la comunidad migrante y el centro de salud» (AS, CESFAM).

TS: Trabajador de Salud; AS: Autoridad/Referente de migración; CESFAM: Centro de Salud Familiar; FI: Facilitador intercultural.

\section{CONTEXTO PARA LA INCORPORACIÓN}

El aumento de población haitiana desafió a los equipos de todos los niveles de atención de salud, con barreras culturales e idiomáticas que dificultaban la comunicación efectiva entre usuarios haitianos y equipo de salud.

«El estrés que producía en los funcionarios era alto, la angustia de no poder comunicarse (...) de no poder explicarle lo que necesitaba, eso producía gran estrés en los funcionarios. Por esa razón se tomó la decisión» (AS, hospital).
Es así como, se incorporó la figura de FI para MI sin perfil específico. Para la selección del FI no fue obligatorio, por ejemplo, contar con formación académica en salud. Se valoraron más las habilidades blandas, concepto que se refiere a las cualidades, características o competencias personales de las personas, relacionadas con la perspectiva emocional y social para desenvolverse y que en la actualidad son altamente valoradas en el mundo laboral, como: comunicación efectiva trabajo en equipo, relaciones interpersonales positivas y capacidad de adaptación al entorno y al equipo de salud; 
por sobre otro tipo de competencias cognitivas, para dar una acogida, orientación y contención al Ml en todo momento en su experiencia en el centro de salud.

"La intención era que las personas pudiesen entregar una información que a la vez estuviera el componente de contención emocional, que fuesen personas preparadas, con herramientas mínimas para resolver situaciones de malas noticias» (AS, hospital).

Tanto TS como FI reconocieron una ventaja comparativa el tener formación académica en salud, pues así contaban con un manejo más acabado de los programas de salud en Chile y podían orientar a los usuarios usando terminología médica. Sin embargo, los FI sin formación en salud manifiestan que sortearon estas carencias formativas consultando oportunamente al equipo de salud.

"Que él tenga esos conocimientos nos facilita la forma de explicar como ellos [MI] van accediendo a atenciones» (TS, CESFAM).

«Hablaban con términos médicos (...) cuando yo no entendía algo, pregunté (...) ellos tratan de decirlo de una manera más simple para que pueda entender» (FI, hospital).

En todos los centros de salud en que se realizó el estudio, los FI para MI tenían un vínculo directo con el empleador y una relación contractual de pago por honorarios. Como contexto, en Chile se establecen contrataciones de personal directo con la empresa en modalidad de planta, plazo fijo y honorarios; y en relación contractual indirecta existe el pago por servicios, en donde una empresa externa es la que contrata al personal que deberá ejercer funciones en el centro de salud.

\section{FUNCIONES}

Fueron reconocidas como funciones del FI por los cuatro grupos de actores clave: la acogida, traducción, orientación y educación del sistema de salud chileno. La acogida tiene relación con la recepción del Ml tanto al centro de salud como a un sistema sanitario distinto al de su país de origen; la traducción está asociada a llevar indicaciones y preguntas de ambas partes (TS y MI) del español al creol y viceversa; la orientación es guiar al Ml sobre la ubicación física de distintos servicios y de cómo «moverse» dentro del sistema de salud chileno, estando directamente relacionado. Finalmente, la educación del sistema sanitario que hacen a la llegada del migrante al centro de salud, más aún en atención primaria.

"Cuando entro al control con el doctor, es él [FI] quien me explica, me acompaña, si yo necesito medicamentos él desciende conmigo abajo y después él sube y vuelve a acompañarme a cada lado que debo ir, él siempre está al lado mío» (MI, hospital).

Los MI, a diferencia de los otros actores clave, no mencionan como funciones del FI la interpretación, promoción de salud, mediación cultural y administrativa. Este grupo nombra principalmente funciones relacionadas a la traducción y orientación sobre qué hacer y cómo funciona la red de salud. La función de interpretación, muy valorada por los otros tres grupos, es sumar a la traducción el factor cultural y propio del lenguaje para entender en un contexto lo que se quiere comunicar.

"La facilitadora no sólo tiene que hacer una labor de interpretación, sino que también de ir generando esta comunicación que sea lo más empática y asertiva posible (...) y por otro lado entregar información a medida que lo solicita al equipo de salud» (AS, hospital).

En cuanto a la mediación cultural, es el apoyo que realiza el FI ante diferencias culturales entre usuarios haitianos y TS, enfatizando, por ejemplo, el aporte que realizan para comprender acciones y/o formas de cuidado de madres haitianas con sus hijos. Asociada a esta función está la promoción de la salud, en donde se destaca la participación de los $\mathrm{Fl}$ en actividades de prevención y promoción de salud, tales como talleres: prenatales y a personas insulino dependientes.

"Los profesionales me preguntan: ¿es normal esto? Porque en Haití una vez que nace el bebe no se alimenta con leche materna, sino que, con comida, yo le dije que sí (...) Acá en Chile debemos trabajar con la comunidad, con las madres para poder enseñar como deben cambiar esta conducta, cual es la consecuencia, porqué debe hacerlo así» (FI, CESFAM).

Los TS y las AS destacan la función de enlace con la comunidad, viéndola como una oportunidad de acercarse y conocer más a la población migrante haitiana que consulta, para lograr mayor adherencia tanto al tratamiento y a la red de salud que los acoge. Finalmente, la función administrativa, reconocida por AS y $\mathrm{FI}$, que ha sido un aprendizaje continuo para responder a las demandas de todas las personas, independiente de su nacionalidad.

«Han aprendido a tomar reclamos, las leyes, los derechos, deberes (...) han aprendido tanto del proceso de la atención hospitalaria, diagnósticos, confidencialidad de los diagnósticos, a cómo tienen que informar algunas cosas» (AS, hospital).

\section{VENTAJAS Y DESVENTAJAS DEL FI}

Las ventajas mencionadas por todos los actores clave son, por un lado, la confianza en la comunicación efectiva, $y$, por otro, el uso eficiente de la red de salud, que está directamente abordado por las funciones de traducción, orientación y acogida.

Por otra parte, AS y TS mencionan una percepción de disminución del gasto público al evitar la cronicidad y nuevas hospitalizaciones de usuarios MI que podrían existir de no haber FI y, como consecuencia, no se comprendieran y siguieran correctamente las indicaciones médicas. Además, la reducción de estrés del equipo de salud al poder tener una comunicación efectiva con los usuarios, seguros de que se está realizando una atención de salud con pertinencia intercultural y con respeto al derecho de confidencialidad de diagnóstico de cada usuario. Finalmente, destacan la 
contribución que han realizado los $\mathrm{FI}$, junto a la población migrante en general, para asentar la necesidad de capacitación en temáticas de interculturalidad en salud.

"Una persona que no entiende que le está pasando y por lo tanto, se le entrega una receta y no sabe que esa receta tienen determinadas indicaciones, sino sigue ese tratamiento, lo más probable es que igual se agrave su problema y no solamente le afecte a él, sino que ha toda su familia, a todo su entorno, al Estado que va a tener que tomar otras medidas y también hacer otra inversión en salud de algo que se podría haber atendido en una etapa inicial» (AS, hospital).

"Si no hay facilitador, ella no puede decir lo que siente, aunque quiera decir lo que siente en su idioma, el doctor no va a entender porque el doctor no habla el idioma creol (...). Por eso es importante que cuando una persona aún no habla español, haya un asistente que hable el idioma» (MI, CESFAM).

Al consultar desventajas, ninguno de los entrevistados declara, sólo mencionan barreras administrativas y económicas para su inserción como una limitante a considerar y mejorar a futuro.

\section{BARRERAS Y FACILITADORES PARA INCORPORAR AL FI}

Los TS y AS mencionan barreras de tipo administrativas y económicas para la incorporación formal de esta figura a los equipos de salud, pues no existen experiencias previas a nivel nacional y el foco financiero actual ha estado centrado en atención primaria. A nivel hospitalario, se declara que no hay avances normativos desde la política pública.

«Nunca entendieron que las personas que necesitaban también atención eran aquellos que llegaban a este hospital para que se les informara que tenían que ser operados, que su enfermedad era irreversible» (AS, hospital).

Como facilitadores destacan dos grandes componentes, el primero tiene relación con la voluntad de la autoridad local para la incorporación del FI, sobreponiéndose a barreras administrativas-económicas, y la segunda tiene relación a que en todos los centros tienen credencial de identificación, y uno de ellos ha sumado uniforme institucional.

«El director quería que este hospital fuera un hospital sin muros, donde eran todos bienvenidos» (AS, hospital).

«Tienen uniforme institucional, credencial, el hospital sabe que está presente» (TS, hospital).

\section{RECOMENDACIONES}

Las funciones y la alta demanda hacen que el FI sea considerado un recurso necesario y escaso. Sin embargo, se menciona la necesidad de evaluación de esta figura para su incorporación según la realidad local de los centros de salud que aún no cuentan con este recurso humano. Además, se recomienda potenciar la sensibilización a equipos de salud en temas de intercultural en salud e incorporar al FI en reuniones de sector y estudios de familia en casos de especial cuidado, de manera de contribuir en el análisis de cómo abordar ciertos casos de mayor complejidad con foco intercultural.

«No tiene que ser solamente para traducir, tienen que incorporarse con el equipo y ser de ayuda como un nexo entre la comunidad migrante y el centro de salud» (AS, CESFAM).

"Capacitarnos más a nosotras como profesionales de salud (...) porque las barreras culturales se quitan solamente con la educación» (TS, hospital).

Finalmente, un tema emergente es la necesidad de cuidados de salud mental para los $\mathrm{FI}$, pues están expuestos a situaciones complejas comunicando resultados tales como muertes fetales, cáncer, infecciones de transmisión sexual y $\mathrm{VIH} /$ sida, entre otros. Si bien los FI valoran el apoyo que reciben por parte de los TS, es necesario que reciban apoyo psicoemocional y herramientas de autocuidado formales y permanentes durante la ejecución del rol. Sólo un centro de salud investigado tomó medidas, realizando una reunión semanal moderada por trabajadora social para contención de los $\mathrm{Fl}$, pero otros centros de salud reconocen que esta es una debilidad que requiere urgente atención de los equipos y del sistema de salud en su conjunto.

"No sé cómo lo hacen los demás facilitadores cuando le toca algo complejo, lo único es que a mí a veces me pasa es tener un dolor de cabeza que no se me pasa» (Fl, hospital).

\section{DISCUSIÓN}

Esta investigación sugiere que el Fl es esencial para superar barreras idiomáticas (principal motivo de contratación) y culturales existentes en la atención a población MI haitiana en los centros de salud, situación descrita previamente por Cabrera et al (25). Pero, este estudio documenta que el FI no sólo «traduce», sino que sus funciones son amplias, destacándose conforme al relato de actores clave la mediación cultural, educación del sistema sanitario e interpretación de procesos de salud y enfermedad y de alternativas terapéuticas disponibles. Además, la existencia del FI es considerada una buena práctica en Chile ${ }^{(18)}$ y a nivel internacional ${ }^{(11,12,26)}$. La evidencia propone que contar con servicios profesionales, en vez de familiares o personas voluntarias, para el rol del FI tiene ventajas significativas, puesto que quienes cumplen esta función de manera informal generalmente no tienen habilidades interculturales establecidas, en algunos casos ocultan información y no manejan terminología médica, resultando una atención médica insatisfactoria ${ }^{(5,6)}$.

Los actores clave señalaron que no era un requisito obligatorio para los FI la formación académica en salud, pero sí destacan beneficios que la persona maneje los programas de salud disponibles en el país y terminología médica empleada en la atención sanitaria. Según evidencia internacional, los facilitadores deberían tener, como mínimo, entrenamiento 
en mediación intercultural ${ }^{(7,8)}$, pues la interpretación de indicaciones médicas es un trabajo delicado, donde los profesionales de salud pueden exponerse a mayor riesgo médico legal ${ }^{27)}$. La disminución del gasto público es internacionalmente reconocida como ventaja de contar con FI, pues las asignaciones de intérpretes permiten un tratamiento más efectivo y específico, evitando cronicidad, mejora la adherencia y promueve la satisfacción usuaria ${ }^{(10,28,29)}$.

El reconocimiento del valor y ventajas comparativas de brindar una atención con $\mathrm{FI}$, fueron ampliamente detalladas en este estudio, y también descrito por la literatura como una estrategia relevante de salud pública ${ }^{(7,28)}$. Se destaca como tema emergente de esta investigación la salud mental de los $\mathrm{FI}$, pues se reconoce que este rol puede actuar como un riesgo laboral si no es bien definido, entrenado y apoyado, reforzando la importancia de formación temprana en este tema ${ }^{(30)}$.

A raíz de estos resultados se puede revisar la oportunidad que ofrece para los sistemas de salud el garantizar financiamiento gubernamental de servicios profesionales de traducción e interpretación en la atención médica, con normativas actualizadas y basadas en la evidencia, que abarquen todos los niveles de atención de salud, demanda que quedó expuesta en este estudio para formalizar el financiamiento en nivel secundario. Esto persiste como un desafío para Chile, para países de la región de Latinoamérica y para todos los países del mundo $(4,11,28)$.

La principal fortaleza de esta investigación fue haber realizado entrevistas a cuatro grupos de actores clave involucrados en temática de facilitación lingüística e intercultural hacia población MI. Con ello, se logró un valioso acercamiento a la percepción del rol de los FI en la atención de salud de MI, que consideró la voz de usuarios haitianos. Una limitación del estudio es que, al haberse basado en entrevistas y no en observaciones directas, solamente se obtuvo información de las percepciones de actores clave y no del rol clínico en la atención directa a población MI. Por ello, se requieren de nuevos estudios que permitan observación directa de prácticas interculturales de FI y equipos de salud.

Se concluye que la percepción del rol de los facilitadores está relacionada con funciones específicas en la traducción, interpretación, educación del sistema sanitario, mediación intercultural y colaboración con labores administrativas. Además, colabora en actividades educativas para migrantes que requieren apoyo con el abordaje de diferencias culturales. Tiene importantes ventajas en prestar una atención de salud con pertinencia cultural y respetando la privacidad en la atención sanitaria, por lo que urge regular en materia administrativa-financiera para su incorporación formal en los centros de salud. El reconocimiento de la importancia del FI en la atención en salud con pertinencia intercultural hacia población MI en la Política de Salud para Migrantes Internacionales en Chile es un avance social y del sistema de salud, que puede ser replicado a otros países que enfrentan desafíos similares.

Contribuciones de los autores: CS participó en la concepción y diseño del artículo, recolección de resultados, análisis e interpretación de datos, redacción del artículo, revisión crítica del artículo y aprobación de la versión final. BC participó en la concepción y diseño del artículo, brindó asesoría técnica, redacción del artículo, revisión crítica del artículo y aprobación de la versión final.

Fuentes de financiamiento: Financiamiento parcial de la Comisión Nacional de Investigación Científica y Tecnológica de Chile, mediante Beca Magíster Nacional/2018-22180042.

Conflictos de interés: Las autoras declaran no tener conflictos de interés.

\section{REFERENCIAS BIBLIOGRÁFICAS}

1. Servicio de Salud Metropolitano Central. Guía para los equipos de salud en la orientación y apoyo a la población migrante contenido. Santiago, Chile: Ministerio de Salud; 2015.

2. Cabieses B, Bernales M, McIntyre AM. La migración internacional como determinante social de la salud en Chile: evidencia y propuestas para políticas públicas. $1^{\text {a }}$ ed. Santiago, Chile: Universidad del Desarrollo; 2017.

3. Divi C, Koss RG, Schmaltz SP, Loeb JM. Language proficiency and adverse events in US hospitals: A pilot study. Int J Qual Heal Care. 2007;19(2):60-7.

4. Brisset C, Leanza Y, Laforest K. Working with interpreters in health care: A systematic review and meta-ethnography of qualitative studies. Patient Educ Couns. 2013;91(2):131-40. doi: 10.1016/j. pec.2012.11.008.

5. KrupicF,Hellström M, Biscevic M,SadicS, Fatahi N. Difficulties in using interpreters in clinical encounters as experienced by immigrants living in Sweden. J Clin Nurs. 2016;25(11-12):1721-8. doi: 10.1111/ jocn.13226.

6. Zendedel R, Schouten BC, van Weert JCM, van den Putte B. Informal interpreting in general practice: Comparing the perspectives of general practitioners, migrant patients and family interpreters. Patient Educ Couns. 2016;99(6):981-7. doi: 10.1016/j.pec.2015.12.021.
7. Alcaraz Quevedo M, Paredes-CarbonellJJ, Sancho Mestre C,Lopez-Sanchez P, Garcia Moreno JL, Vivas Consuelo D. Atención a mujeres inmigrantes en un programa de mediación intercultural en salud. Rev Esp Salud Publica. 2014;88(2):301-10. doi: 10.4321/S1135-57272014000200012.

8. Hadziabdic E, Heikkilä K, Albin B, Hjelm K. Migrants' perceptions of using interpreters in health care. Int Nurs Rev. 2009;56(4):461-9. doi: 10.1111/j.14667657.2009.00738.x.

9. Morina N, Maier T, Schmid Mast M. Lost in translation?--psychotherapy using interpreters. Psychother Psychosom Medizinische Psychol. 2010;60(3-4):10410. doi: $10.1055 / \mathrm{s}-0029-1202271$. 
10. Schreiter S, Winkler J, Bretz J, SchoulerOcak M. Was kosten uns Dolmetscher? - Eine retrospektive Analyse der Dolmetscherkosten in der Behandlung von Flüchtlingen in einer Psychiatrischen Institutsambulanz in Berlin. PPmP Psychother Psychosom Medizinische Psychol. 2016;66(9-10):356-60. doi: 10.1055/s-0042-115414.

11. Taglieri FM, Colucci A, Barbina D, Fanales-Belasio E, Luzi AM. Communication and cultural interaction in health promotion strategies to migrant populations in Italy: the cross-cultural phone counselling experience. Ann Ist Super Sanità. 2013;49(2):138-42. doi: 10.4415/ANN_13_02_05.

12. Hadziabdic E, Albin B, Heikkilä K, Hjelm K. Family members' experiences of the use of interpreters in healthcare. Prim Health Care Res Dev. 2014;15(2):156-69. doi: $10.1017 /$ S1463423612000680.

13. Instituto Nacional de Estadisticas, Departamento de Extranjería y Migración. Estimación de Personas Extranjeras Residentes en Chile. 31 de Diciembre de 2018 [Internet]. Santiago: Instituto Nacional de Estadisticas; 2019 [citado el 22 de octubre de 2019]. Disponible en: https://ine.cl/docs/default-source/ default-document-library/estimaciónde-personas-extranjeras-residentesen-chile-al-31-de-diciembre-de-2018. pdf ?sfvrsn $=0$

14. Instituto Nacional de Estadisticas. Resultados CENSO 2017 [Internet].Santiago: Instituto Nacional de Estadisticas; 2018 [citado el 22 de octubre de 2019]. Disponible en: https:// resultados.censo2017.cl/Home/Download

15. SEREMI Región Metropolitana. Diagnóstico de Salud de Inmigrantes Región Metropolitana. Santiago de Chile: Ministerio de Salud; 2015.

16. Van der Laat C. La migración como determinante social de la salud. En: Cabieses
B, Bernales M, McIntyre AM, editors. La migración internacional como determinante social de la salud en Chile: evidencia y propuestas para políticas públicas. $1^{a} \mathrm{ed}$. Santiago de Chile: Universidad delDesarrollo; 2017.p.29-38.

17. Bernales M, Cabieses B, McIntyre AM, Chepo M. Desafíos en la atención sanitaria de migrantes internacionales en Chile. Rev Peru Med Exp Salud Publica. 2017;34(2):167-75. doi: 10.17843/ rpmesp.2017.342.2510.

18. Ministerio de Salud de Chile, FONASA, Superintendencia de Salud. Política de Salud de Migrantes Internacionales en Chile. Santiago de Chile: Ministerio de Salud; 2017.

19. Comisión de Política de Salud y Pueblos Indígenas. Política de Salud y Pueblos Indígenas. Santiago de Chile: Ministerio de Salud; 2003.

20. Ministerio de Salud, Banco Mundial. Plan para Pueblos Indígenas. Proyecto de Apoyo al Sector Salud. Santiago de Chile: Ministrio de Salud; 2017.

21. Sánchez P. K, Valderas J. J, Messenger C. K, Sánchez G. C, Barrera Q. F, Sánchez P. K, et al. Haití, la nueva comunidad inmigrante en Chile. Rev Chil Pediatr. 2018;89(2):278-83. doi: 10.4067/S037041062018000200278.

22. Martínez Carazo PC. El método de estudio de caso Estrategia metodológica de la investigación científica. Pensamiento \& Gestión. 2006;20:165-93.

23. Batthyány K, Cabrera M. Metodología de la investigación en Ciencias Sociales: apuntes para un curso inicial. Metodologia de investigacion en Ciencias Sociales. Montevideo: Universidad de la República; 2011.

24. Sandoval Casilimas CA. Investigación cualitativa. $1^{\text {a }}$ ed. Bogotá Colombia:
Instituto Colombiano para el Fomento de la Educación Superior; 2000.

25. Cabrera Cuevas Y, Blanco Nuñez G, Ramos Barrios D. Estudio de caso: comuna de Quilicura. En: Cabieses B, Bernales M, McIntyre AM, editors. La migración internacional como determinante social de la salud en Chile: evidencia y propuestas para políticas públicas. $1^{\text {a }}$ ed. Santiago de Chile: Universidad del Desarrollo; 2017. p. 471-84.

26. Liu P, Guo Y, Qian X, Tang S, Li Z, Chen L. China's distinctive engagement in global health. Lancet. 2014;384(9945):793-804. doi: 10.1016/S0140-6736(14)60725-X.

27. Gray B, Hilder J, Donaldson H. Why do we not use trained interpreters for all patients with limited English proficiency? Is there a place for using family members? Aust J Prim Health. 2011;17(3):240-9. doi: 10.1071/PY10075.

28. Iniesta C, Sancho A, Castells X, Varela J. Hospital orientado a la multiculturalidad. Experiencia de mediación intercultural en el Hospital del Mar de Barcelona. Med Clin. 2008;130(12):472-5. doi: $10.1157 / 13118113$

29. Beach MC, Price EG, Gary TL, Robinson KA, Gozu A, Palacio A, et al. Cultural competence: A systematic review of health care provider educational interventions. Med Care. 2005;43(4):356-73.

30. De Muynck A. Mediación intercultural: ¿una herramienta para promover la salud de las inmigrantes? Quadem CAPS. 2004;32:40-6.

\footnotetext{
Correspondencia: Camila Sepúlveda

Dirección: Av. Las Condes 12461, Las Condes, Región Metropolitana, Santiago, Chile. Teléfono: +56225785534

Email: csepulvedaastete@gmail.com
} 